\title{
Dynamic Multi-Depot Multi-Compartment Refrigerated Vehicle Routing Problem with Multi-Path Based on Real-Time Traffic Information
}

\author{
Dengkai HOU, Houming FAN and Xiaoxue REN ${ }^{1}$ \\ College of Transportation Engineering, Dalian Maritime University, Dalian 116026, \\ China
}

\begin{abstract}
Aiming at the dynamic multi-depot multi-compartment refrigerated vehicle routing problem with multi-path based on real-time traffic information, based on the idea of pre-optimization followed by real-time adjustment, a two stages optimization model with the goal of minimizing total cost is established. To solve this problem, this paper designed a hybrid chaotic genetic algorithm with variable neighbourhood search (HCGAVNS) to generate the initial routes. In the real-time adjustment phase, this paper proposed a path selection strategy to update the selected paths. Multiple experiments are constructed to verify the validity of the model and the algorithm. This research has important theoretical and practical significance.
\end{abstract}

Keywords. Real-time traffic information, multi-path, hybrid chaotic genetic algorithm with variable neighborhood search.

\section{Introduction}

Dynamic multi-depot multi-compartment refrigerated vehicle routing problem with multi-path based on real-time traffic information is a research based on multi-depot joint delivery and real-time traffic information, with considering the multicompartment refrigerated vehicle and multi-path between nodes. The vehicle departs from any depot, and in the delivery process, the vehicle can adjust the selected path in real time according to the influence of road traffic conditions on the vehicle speed to minimize the cargos damage cost and delivery time. Multi-compartment refrigerated vehicles can meet the transportation of fresh products with different temperature requirements at the same time. In recent years, it has been widely used in the transportation of fresh products such as seafood and vegetables.

The contributions of this paper are as follows:

(1) Based on real-time traffic information, the idea of pre-optimization followed by real-time adjustment is proposed.

\footnotetext{
${ }^{1}$ Corresponding Author, Xiaoxue REN, College of Transportation Engineering, Dalian Maritime University, Dalian 116026, China; Email: rxx1587351294@163.com.
} 
(2) The hybrid chaotic genetic algorithm with variable neighbourhood search is designed considering the influence of multi-compartment refrigerated vehicle, multipath, customer time window and real-time update of vehicle speed.

\section{Literature Review}

This paper reviews the literature of dynamic vehicle routing problem based on realtime traffic information and multi-path vehicle routing problem.

For dynamic vehicle routing problem based on real-time traffic information, Kok et al. [1] proposed some strategies to avoid traffic congestion, such as choosing alternative routes, changing the order of customer visits, changing the vehicle-customer allocation, and combining Dijkstra algorithm with heuristic algorithm to solve the problem. Sabar et al. [2] proposed an adaptive evolutionary algorithm to solve the dynamic vehicle routing problem with traffic congestion, and multiple experiments are constructed to verify the proposed algorithm. Jin et al. [3] established a bi-objective model with considering the economic and environmental objectives simultaneously, and a nearest neighbour-first iterated local search-second algorithm is designed to solve it. Rozas et al. [4] proposed a predictive decision strategy using historical and real-time traffic data to solve the dynamic stochastic shortest path problem of electric vehicles in real time. Kritzinger et al. [5] proposed an experimental evaluation to solve the timedependent vehicle routing problem with real-world traffic information.

For vehicle routing problem with multi-path, Garaix et al. [6] first applied multipath to vehicle routing problem (VRP) and designed a dynamic programming solution method to solve it. Ticha et al. [7] designed an adaptive large neighbourhood search (ALNS) heuristic to solve the vehicle routing problem with multi-path. Wang et al. [8] established a mathematical model to minimize the sum of vehicle dispatch cost, time cost and transportation cost, and a particle swarm optimization with local improvement to solve the vehicle routing problem with multi-path. Huang et al. [9] established two time-dependent vehicle routing problem with path flexibility models under deterministic and stochastic traffic conditions, and improved Dijkstra algorithm is designed to solve it. Setak et al. [10] modelled the problem as a time-dependent vehicle routing problem with multi-path and FIFO characteristics. In addition, a heuristic tabu search (TS) algorithm is proposed to solve it.

\section{Problem and Mathematical Model}

\subsection{Problem Description}

The problem is described as follows: the delivery network has a complete directed graph $G=(V, E)$, where nodes set is $V=V_{0} \cup V_{1}, V_{0}$ represents the depot set, $V_{1}$ represents the customer set. This paper assume there are multiple paths between two nodes, $m$ represents any path in the path set $M$ between two nodes, and the vehicle only chooses one of them to travel. The distance of path $m$ between node $i$ and node $j$ is $l_{i j m} . k$ represents a vehicle and each vehicle has a refrigerated compartment and a frozen compartment with capacity $Q_{h}$, which $h \in H, H$ represents the product set, 
and $h=1$ represents the refrigerated cargo, $h=2$ represents the frozen cargo. The demand of customer $i$ for each product is $d_{i h}$. [T $\left.T_{s}, T_{f}\right]$ is the depot time window and $\left[E T_{i}, L T_{i}\right]$ is the customer time window. $c_{1}$ represents the waiting cost per unit time and $c_{2}$ represents the delay cost per unit time. $c_{3}$ represents the price of fuel, $c_{4}$ represents the vehicle dispatched cost. $t_{i}^{s}$ represents the service time at customer $i$. $x_{i j k m}$ indicates whether vehicle $k$ travels from node $i$ to node $j$ through path $m$.

\subsection{Speed Function Determination}

This paper predicts the vehicle travel speed of each road section based on the historical traffic information obtained from the big data platform. The day is divided into $P$ periods with a period of 30s. The time $t_{i j m}$ that vehicle travels from node $i$ to node $j$ through path $m$ has two possibilities of inter-period and intra-period, and is calculated as in equation (1).

$$
t_{i j m}=\left\{\begin{array}{l}
l_{i j m} / v_{p m}, l_{i j m} \leq\left(T_{p}-T_{i k}\right) v_{p m} \\
\sum_{f \in F} l_{i j m(p+f)} / v_{m(p+f)}, \text { otherwise }
\end{array}\right.
$$

\subsection{Refrigeration Cost and Cargo Damage Cost}

To maintain the temperature of the carriage, refrigerant is consumed. Reference [11] identifies the following method for calculating refrigeration costs.

$$
C_{\text {cold }}=\sum_{i \in V} \sum_{i \in V} \sum_{m \in M} \sum_{k \in K}\left[\left(\rho \sqrt{s_{1} s_{2}} \Delta T_{1} \alpha+\rho \sqrt{s_{3} s_{4}} \Delta T_{2} \alpha\right) t_{i j m} c_{5} x_{i j k m}\right]+\sum_{i \in V} \sum_{j \in V_{1}} \sum_{k \in K} \sum_{m \in M} t_{i}^{s} c_{6} x_{i j k m}
$$

Where, $\Delta T_{1}$ and $\Delta T_{2}$ represent the internal and external temperature difference of the refrigerated and frozen areas respectively. $\rho$ represents the thermal conductivity the vehicle. $\alpha$ is the thermal load coefficient during loading and unloading. $s_{1}$ and $s_{2}$ represent the external and internal surface areas of the refrigerated carriage. $s_{3}$ and $s_{4}$ represent the external and internal surface areas of the frozen carriage. $V$ is the compartment volume. $c_{5}$ indicates the refrigerant unit price. $c_{6}$ is refrigeration cost per unit time during loading and unloading. Fresh products after the impact of factors such as vehicle body shaking, loading and unloading, cargo damage will occur. The cargo damage cost is calculated as follows.

$$
\begin{aligned}
C_{\text {damage }} & =\varphi_{1} \sum_{i \in V} \sum_{j \in V} \sum_{k \in K} \sum_{m \in M} \sum_{h \in H} c_{7}\left[t_{i j m} Q_{i h}+t_{j}^{s}\left(Q_{i h}-d_{j h}\right)\right] x_{i j k m} \\
& +\varphi_{2} \sum_{i \in V} \sum_{j \in V} \sum_{k \in K} \sum_{m \in M} \sum_{h \in H} c_{8}\left[t_{i j m} Q_{i h}+t_{j}^{s}\left(Q_{i h}-d_{j h}\right)\right] x_{i j k m}
\end{aligned}
$$

Where, $c_{7}, c_{8}$ denotes the value per unit of refrigerated and frozen cargo respectively. $\varphi_{1}, \varphi_{2}$ denotes the loss rate of cargo in refrigerated compartments and in 
frozen compartments respectively, determined by the Arrhenius equation[12]. $Q_{i h}$ is the load in compartment $h$ when the vehicle leaves node $i$.

\subsection{Mathematical Model}

To solve the problems, the strategy of "pre-optimization followed by real-time adjustment " is adopted.

(1) Pre-optimization stage model

$$
\begin{aligned}
\min C= & c_{1} \sum_{i \in V} \sum_{j \in V_{1}} \sum_{k \in K} \sum_{m \in M} x_{i j k m} \max \left\{\left(E T_{j}-T_{j k}\right), 0\right\}+ \\
& c_{2} \sum_{i \in V_{1}} \sum_{j \in V} \sum_{k \in K} \sum_{m \in M} x_{i j k m} \max \left\{\left(T_{i k}-L T_{i}\right), 0\right\}+ \\
& c_{3} \sum_{i \in V} \sum_{j \in V} \sum_{k \in K} \sum_{m \in M} l_{i j m} x_{i j k m}+c_{4} \sum_{i \in V_{0}} \sum_{j \in V_{1}} \sum_{k \in K} x_{i j k}+C_{\text {cold }}+C_{\text {damage }}
\end{aligned}
$$

s.t.

$$
\begin{gathered}
\sum_{j \in V_{1}} \sum_{m \in M} \sum_{k \in K} x_{i j k m} \leq|K|, \forall i \in V_{0} \\
\sum_{m \in M} \sum_{i \in V_{0}} \sum_{j \in V_{1}} x_{i j k m}=\sum_{m \in M} \sum_{j \in V_{1}} \sum_{i \in V_{0}} x_{j i k m} \leq 1, \forall k \in K \\
\sum_{m \in M} \sum_{k \in K} \sum_{i \in V} x_{i j k m}=\sum_{m \in M} \sum_{k \in K} \sum_{i \in V} x_{j i k m}=1, \forall j \in V_{1} \\
\sum_{m \in M} \sum_{i \in V} \sum_{j \in V_{1}} \sum_{h \in H} x_{i j k m} d_{i h} \leq Q_{h} \\
\sum_{m \in M} \sum_{i \in S} \sum_{j \in S} x_{i j k m} \leq|S|-1, \forall k \in K \\
T_{s}+\sum_{i \in V} \sum_{j \in V} \sum_{m \in M} x_{i j k m} t_{i j m}+\sum_{i \in V} \sum_{j \in V_{1}} \sum_{m \in M} t_{j}^{s} x_{i j k m} \leq T_{f}, \forall k \in K \\
\left(T_{i k}+t_{i}^{s}+t_{i j k m}\right) x_{i j m} \leq T_{j k}, \forall(i, j) \in V_{1}, \forall k \in K, \forall m \in M \\
x_{i j k m}=\{0,1\}, \quad \forall i \in V, \forall j \in V, \forall k \in K, \forall m \in M
\end{gathered}
$$

Eq. (4) is the objective function to minimize the total cost. Eq. (5) indicates that vehicles dispatched from the depot cannot exceed the maximum number of vehicles. Eq. (6) indicates a vehicle has only one route, and is the entry and exit equilibrium constraint. Eq. (7) indicates that each customer is served only once. Eq. (8) indicates that the demand for each product by each vehicle serving customers does not exceed the capacity of each compartment. Eq. (9) is the subtour elimination constraint. Eq. (10) indicated that the vehicle must return to the depot before the working deadline. Eq. (11) calculates the arrival time of the vehicle from node $i$ to node $j$. Eq. (12) defines the attributes of the decision variable.

(2) Real-time adjustment strategy and model.

In the real-time adjustment stage, the order of customers in the pre-optimization stage is kept unchanged, and the actual arrival time is calculated by adding the travel time through the real-time path and the service time of previous node together. $t_{i j m}^{z}$ indicates 
the actual time that the vehicle travels from node $i$ to node $j$ through path $m . T_{i k}^{z}$ indicates the actual time that vehicle $k$ arrives at node $i$.

$$
\begin{aligned}
\min C= & c_{1} \sum_{i \in V} \sum_{j \in V_{1}} \sum_{k \in K} \sum_{m \in M} x_{i j k m} \max \left\{\left(E T_{j}-T_{j k}\right), 0\right\}+ \\
& c_{2} \sum_{i \in V_{1}} \sum_{j \in V_{1}} \sum_{k \in K} \sum_{m \in M} x_{i j k m} \max \left\{\left(T_{i k}-L T_{i}\right), 0\right\}+ \\
& c_{3} \sum_{i \in V} \sum_{j \in V} \sum_{k \in K} \sum_{m \in M} l_{i j m} x_{i j k m}+C_{\text {cold }}+C_{\text {damage }}
\end{aligned}
$$

s.t.

Eqs. (5)-(10), (12)

$$
\left(T_{i k}^{z}+t_{i}^{s}+t_{i j m}^{z}\right) x_{i j k m} \leq T_{j k}^{z}, \forall(i, j) \in V_{1}, \forall k \in K, \forall m \in M
$$

Eq. (14) ensures that the real moment of arrival of the customer must meet the real travel time between customers.

\section{Solution Approach}

In this paper, an improved hybrid chaos genetic algorithm with variable neighbourhood search algorithm (HCGAVNS) is designed considering the advantages and disadvantages of genetic algorithm and variable neighbourhood search algorithm.

\subsection{Encoding and Initial Population Generation}

Integer coding is adopted in this paper. In this paper, the pseudo-randomness of chaotic system is used to generate initial population by Logistics mapping equation, as shown in equation (15).

$$
x_{n+1}=r x_{n}\left(1-x_{n}\right), n=1,2, \cdots, r \in(3.57,4], x_{i} \in[0,1]
$$

When $r=4$, the system is in a state of complete chaos, and it has all the properties of chaotic systems.

\subsection{Fitness}

The fitness value in this paper is the reciprocal of the value of the objective function, as shown in equation (16).

$$
f=1 / C
$$

\subsection{Selection}

This paper adopts the strategy of elite reservation and roulette to select individuals. Firstly, the elite reservation strategy is adopted to reserve some optimal individuals. Then, the remaining individuals are selected by roulette strategy. 


\subsection{Local Search Strategy}

In this paper, variable neighbourhood search algorithm is used to enhance the local search ability of the algorithm. Neighbourhood structure sets $N_{k}=\left\{N_{1}, N_{2}, \cdots N_{l}\right\}$ are constructed. Individual $x$ is searched from the first neighbourhood structure $N_{1}$, and starts from the beginning if an improved solution is found; Otherwise, it enters the next neighbourhood structure. This paper adopts three neighbourhood structures to search, respectively: Insert, Exchange and 2-OPT.

\section{Numerical Experiment}

In this paper, the A-n45-k6 standard instance is modified to generate the instance with 4 depots and 44 customers in line with the study in this paper. There are three paths between any two nodes in the distribution network. The distance between two nodes in different paths is different. The cargos are divided into two categories, one is the refrigerated goods, which need the temperature of $0^{\circ} \mathrm{C}$, and the other is the frozen cargos, which need the temperature of $-10^{\circ} \mathrm{C}$. The payload capacity of vehicle is $8 t$, and the compartment is divided into refrigerated compartment and refrigerated compartment according to $2: 1$. Set other parameters as follows: $\left[T_{s}, T_{f}\right]=[6: 00,9: 00]$, $c_{1}=50, c_{2}=100, c_{3}=1.95, c_{4}=150, c_{5}=26, c_{6}=12, c_{7}=6000$, $c_{8}=18000, \alpha=0.14, \rho=0.024$.

\subsection{Pre-Optimization Stage}

In this section, table 1 shows the distribution routes in the pre-optimization phase. There are three paths between any two nodes in the instance, and the third column in the table 1 is the selected path by the vehicle from one node the next node.

As can be seen from table 1, vehicles will choose different paths according to the impact of the distribution network on vehicle speed when passing through any two nodes. Only vehicle 2, vehicle 3 and vehicle 9 return to the original depot after completing the distribution service, while the rest of the vehicles return to any nearby depot after completing the distribution service.

Table 1. Pre-optimization phase distribution routes.

\begin{tabular}{lccccc}
\hline Number & Route & Path & $\begin{array}{c}\text { Delivery time } \\
\text { (hours) }\end{array}$ & Total cost & $\begin{array}{c}\text { Cargo damage } \\
\text { cost }\end{array}$ \\
\hline Vehicle 1 & $2-17-26-11-32-13-27-1$ & $1-2-3-1-2-2-1$ & 3.8830 & 856.43 & 134.67 \\
Vehicle 2 & $2-25-37-45-14-7-2$ & $1-2-2-1-3-2$ & 3.9085 & 765.61 & 108.59 \\
Vehicle 3 & $3-47-28-41-38-34-3$ & $3-3-2-3-3-2$ & 3.9741 & 744.53 & 156.96 \\
Vehicle 4 & $2-24-12-20-8-46-4$ & $1-3-2-3-2-3$ & 2.7024 & 824.30 & 89.25 \\
Vehicle 5 & $3-23-44-15-33-30-1$ & $3-2-2-3-1-2$ & 3.0097 & 666.97 & 120.86 \\
Vehicle 6 & $3-31-10-48-5-39-18-1$ & $3-2-1-1-3-3-1$ & 2.9256 & 697.01 & 140.63 \\
Vehicle 7 & $1-35-36-22-21-3$ & $2-1-1-2-1$ & 4.7623 & 1140.00 & 155.79 \\
Vehicle 8 & $2-9-19-29-40-43-16-6-1$ & $1-2-3-2-2-1-1-2$ & 6.9677 & 1498.10 & 234.71 \\
Vehicle 9 & $1-42-1$ & $2-1$ & 0.3885 & 313.91 & 4.11 \\
& Total & & 32.5218 & 7506.88 & 1145.56 \\
\hline
\end{tabular}




\subsection{Real-Time Adjustment Stage}

In this stage, only the secondary selection of paths is carried out based on preoptimization, namely according to the updated information to select the reasonable path. Table 2 shows the distribution routes in the real-time phase.

It can be seen from table 1 and table 2 that the total delivery time and total cargo damage cost are reduced by $1.64 \%$ and $3.14 \%$, respectively, to meet customers' requirements for the delivery of fresh products to a greater extent.

Table 2. Adjust phase delivery routes in real time.

\begin{tabular}{ccccc}
\hline Number & Routing & Graph & Delivery time (hours) & Freight damage cost \\
\hline Vehicle 1 & $2-17-26-11-32-13-27-1$ & $1-2-3-1-2-2-1$ & 3.8265 & 132.44 \\
Vehicle 2 & $2-25-37-45-14-7-2$ & $1-2-2-1-3-1$ & 3.8321 & 106.26 \\
Vehicle 3 & $3-47-28-41-38-34-3$ & $3-3-2-3-3-1$ & 3.9257 & 154.66 \\
Vehicle 4 & $2-24-12-20-8-46-4$ & $1-3-2-3-2-1$ & 2.5295 & 84.26 \\
Vehicle 5 & $3-23-44-15-33-30-1$ & $3-2-2-3-1-2$ & 3.0123 & 120.56 \\
Vehicle 6 & $3-31-10-48-5-39-18-1$ & $3-2-1-1-3-3-2$ & 2.8981 & 130.60 \\
Vehicle 7 & $1-35-36-22-21-3$ & $2-1-1-2-1$ & 4.5632 & 150.00 \\
Vehicle 8 & $2-9-19-29-40-43-16-6-1$ & $1-2-3-2-2-1-1-2$ & 7.0259 & 226.87 \\
Vehicle 9 & $1-42-1$ & $2-1$ & 0.3737 & 3.95 \\
& Total & & 31.9870 & 1109.60 \\
\hline
\end{tabular}

\section{Conclusions}

The conclusions of this paper are as follows:

(1) The real-time traffic information reflected by big data platform and the characteristics of complex and diverse road network environment in real life are used to plan distribution routes, which is conducive to improving distribution efficiency.

(2) The established model considers the impact of vehicle route planning on the damage degree of fresh goods and the impact of refrigeration cost, vehicle dispatch cost and time window penalty cost on the total cost, which can be more in line with the actual distribution

(3) The HCGAVNS ensures the diversity of initial solutions by using chaos phenomenon and adopts the strategy of elite reservation and roulette to ensure the effective convergence of the algorithm.

\section{Acknowledgments}

This work was supported by the Special Project of National Emergency Management System Construction of the National Social Science Fund of China (20VYJ024).

\section{References}

[1]Kok A L, Hans E W and Schutten J M J 2012 Vehicle routing under time-dependent travel times: the impact of congestion avoidance Computer Operation Research vol 39 pp 910-18. 
[2] Sabar N R, Bhaskar A, Chung E, Turky A and Song A 2019 A self-adaptive evolutionary algorithm for dynamic vehicle routing problems with traffic congestion Swarm \& Evolutionar Computation vol 44 pp 1018-27.

[3] Jin Y, Ge X, Zhang L and Ren J 2021 A two-stage algorithm for bi-objective logistics model of cash-in-transit vehicle routing problems with economic and environmental optimization based on real-time traffic data Journal of Industrial Information Integration p 100273.

[4]Rozas H, Muñoz-Carpintero D, Saéz D and Orchard M E 2021 Solving in real-time the dynamic and stochastic shortest path problem for electric vehicles by a prognostic decision making strategy Expert Systems with Applications vol $184 \mathrm{p}$ 115489.

[5] Kritzinger S, Doerner K F, Hartl R F, Kiechle G Y, Stadler H and Manohar S S 2012 Using Traffic Information for Time-Dependent Vehicle Routing ProcediaSocial and Behavioral Sciences vol 39 no 39 pp 217-229.

[6] Garaix T, Artigues C, Feillet D and Josselin D 2010 Vehicle routing problems with alternative paths: An application to on-demand transportation European Journal of Operational Research vol 204 no 1 pp 62-75.

[7] Ticha H B, Absi N, Feillet D and Quilliot A 2019 Multigraph modeling and adaptive large neighborhood search for the vehicle routing problem with time windows Computers \& operations research vol 104 no 4 pp 113-126.

[8] Wang H F, Lee Y Y 2014 Two-stage Particle Swarm Optimization Algorithm for the Time Dependent Alternative Vehicle Routing Problem Applied \& Computational Mathematics vol 3 no 4 pp 1-9.

[9]Huang Y, Zhao L, Woensel T V and Gross J P 2017 Time-dependent vehicle routing problem with path flexibility Transportation Research Part B vol 95 no 1 pp 169-195.

[10] Setak M, Habibi M, Karimi H, Abedzadeh M 2015 A time-dependent vehicle routing problem in multigraph with FIFO property Journal of Manufacturing Systems vol 35 no 4, pp 37-45.

[11] Hu W, Liang C J, and Fan L B 2016 Optimization of multi-temperature jointdelivery based on simultaneous pickup and delivery Journal of Guangxi University (Natural Science Edition) vol 41 no 5 pp 1576-84.

[12] Liang C J and Zou Q Q 2017 Research on vehicle routing problem with temperature variable in cold chain logistics Journal of Guangxi University (Natural Science Edition) vol 42 no 5 pp 1802-09. 